\title{
CONCEPTUALIZACIÓN HISTÓRICA DEL MARKETING
}

\section{HISTORICAL MARKETING CONCEPTUALIZATION}

\author{
Jácome-Santos Xavier ${ }^{1 *}$; Zambrano-Vera Monica²; Cedeño-García Rossana ${ }^{3}$ \\ ${ }^{1}$ Docente de la Universidad Laica Eloy Alfaro de Manabí, ULEAM. Manta, Ecuador. \\ ${ }^{2}$ Docente de la Universidad Laica Eloy Alfaro de Manabí, ULEAM. Manta, Ecuador. \\ ${ }^{3}$ Analista de la Universidad Laica Eloy Alfaro de Manabí, ULEAM. Manta, Ecuador. \\ *Correo: jasa18@gmail.com
}

\begin{abstract}
Resumen
El presente manuscrito tiene como objetivo analizar los aspectos más relevantes de la conceptualización del marketing, definiéndolo según las etapas propuestas. Bajo este contexto, se define el período preconceptual, período conceptual formal y período actual del concepto de marketing. Además, se proponen las características del marketing, las subdivisiones fundamentales: estrategia y operatividad y se analiza la aplicación de este último. La principal diferencia a resaltar entre el marketing estratégico y el operativo es que la función principal del estratégico es fijar los objetivos a largo plazo mientras que el operativo se basa en acciones concretas a realizar en un plazo medio/corto para lograr los objetivos establecidos. Se analiza el caso puntual de China, que a pesar de ser una potencia mundial aún no desarrolla por completo el marketing estratégico, en contraste con su principal competidor, Estados Unidos.
\end{abstract}

Palabras clave: marketing, estrategia, operativo, conceptos, historia.

\begin{abstract}
This manuscript aims to analyze the most relevant aspects of marketing conceptualization, defining it according to the proposed stages. Under this context, the preconceptual period, formal conceptual period and current period of the marketing concept are defined. In addition, the characteristics of marketing are proposed, the fundamental subdivisions: strategy and operability and the application of the latter is analyzed. The main difference to highlight between strategic and operational marketing is that the main function of the strategic is to set the long-term objectives while the operation is based on concrete actions to be carried out in a medium / short term to achieve the established objectives. The specific case of China is analyzed, which despite being a world power does not yet fully develop strategic marketing, in contrast to its main competitor, the United States.
\end{abstract}

Keywords: marketing, strategy, operational, concepts, history.

Información del manuscrito:

Fecha de recepción: 04 de septiembre de 2019

Fecha de aceptación: 13 de diciembre de 2019

Fecha de publicación: 10 de enero de 2020 


\section{Introducción}

El marketing se define como un conjunto de estrategias empleadas para la comercialización de un producto y para estimular su demanda. Además, tiene como finalidad estructurar un proceso de interacción entre el proveedor y el consumidor de un producto 0 servicio (Lee, 2004).

La Asociación Americana de Marketing, por sus singlas en inglés AMA, propone la siguiente definición para el marketing: "el proceso de planificación y ejecución de la elaboración, fijación de precios, promoción y distribución de ideas, bienes y servicios para crear intercambios que satisfagan los objetivos económicos individuales y organizacionales" (Bennett, 1988).

Webster (1992) señala que el marketing tiene tres dimensiones distintas: como cultura, como estrategia y como táctica. Como cultura, el marketing es un conjunto básico de valores y creencias con respecto a la importancia central del cliente para guiar a la organización. Lambin \& Peeters (1981) expresaron que, pese a que el término marketing se emplea de manera universal, no se establecen acuerdos referentes a su conceptualización y extensión, considerando que los sectores empresariales, de educación y la sociedad en general manejan distintas perspectivas del alcance y aplicación del marketing. Por consiguiente, el estudio del marketing se delimita cronológicamente en períodos que determinaron el origen y evolución del concepto (Coca, 2008).

El plan de marketing estratégico constituye una vía para lograr las metas trazadas de manera óptima y eficiente. A diferencia de la planificación, la estrategia define las pautas para evaluar varias rutas que permitirán, de forma que se obtenga el resultado más adecuado a las necesidades del cliente 0 consumidor (West et al., 2015). Se plantean estrategias para el posicionamiento del marketing estratégico. Los objetivos direccionan las actividades de la empresa, y como tal, objetivos ambiciosos y a largo plazo producen un desarrollo empresarial con mayor sostenibilidad en el mercado. La gran mayoría de las organizaciones corporativas (73\%) se plantean objetivos asociados con mecanismos que permitan potenciar 
las ventas y no con el posicionamiento y mantenimiento en el área comercial (Huan et al., 2008).

Por lo tanto, este trabajo tiene como objeto revisar el origen y desarrollo del marketing, definiendo los períodos de conceptualización, las nuevas tendencias y las características del marketing estratégico.

\section{Historia del marketing}

El marketing es un término con origen anglosajón, aunque es notable que su uso se ha generalizado por todo el mundo, de manera tal que en países de habla hispana se hace uso directo de este término, afirmándose que otros términos no son lo suficientemente amplios como para expresar su significado. Frecuentemente, el marketing se relaciona con diversos significados. En este sentido autores como Kotler \& Amstrong (2003) reportan que la mayoría de personas definen al marketing como un conjunto de actividades que tienen como fin vender y anunciar. En concordancia, el estudio del marketing se delimita cronológicamente en períodos que determinaron el origen y evolución del concepto (Coca, 2008).
Durante los años comprendidos entre 1900 y 1959 se estructura el período preconcenptual. Se reportan investigaciones que proponen definir al marketing como aquella parte de la economía que relaciona la producción de un bien o servicio con el consumidor. Como un acontecimiento importante, se resalta la primera aparición del término marketing en un folleto descriptivo titulado: The distributive and regulative industries of the United States. Por su parte, en 1914 el profesor Weld L. difundió el primer trabajo de investigación científica relacionado con el marketing, en la Asociación Económica Americana (Bartels, 1988).

Posteriormente, surge la fase de conceptualización formal, en el período que inicia en 1960 y finaliza en 1989. La aportación más trascendente la genera la Asociación Americana de Marketing (AMA por sus siglas en inglés). La AMA define al marketing "como la realización de actividades empresariales que dirigen el flujo de bienes y servicios desde el productor hasta el consumidor o usuario". A partir de esta definición, en el año 1964 Edmund McCarthy concluye que "el 
marketing es el resultado de la actividad de las empresas que dirige el flujo de bienes y servicios desde el productor hasta el consumidor 0 usuario, con la pretensión de satisfacer a los consumidores y permitir alcanzar los objetivos de las empresas" (McCarthy, 1964).

Siguiendo esta línea de estudio, la Universidad de Ohio plantea una discusión que sería evaluada por distintos autores, en donde se propone incluir en el marketing los intercambios sociales 0 no lucrativos. Si bien Arndt (1978) sugiere restringir el acceso del marketing al sector no lucrativo, debido a que amplía a sobremanera su extensión; Kotler \& Levy (1969) estiman que la fundamentación del marketing debe ser amplia con alcance sobre las actividades no empresariales, debido a que, en la mayoría de los casos, estas instituciones emplean las variables de la mercadotecnia. Este período se cierra con una nueva aparición de la AMA, en la cual define al marketing de manera menos ambigua, "como el proceso de planificación y ejecución de la creación, precio, comunicación y distribución de ideas, productos y servicios con el fin de crear intercambios que satisfagan a los individuos y a los objetivos de la organización. Consecuentemente, esto genera un espacio a lo que posteriormente se denominaría como marketing social y marketing estratégico.

Desde 1990 se denomina período actual del concepto del marketing, en donde se posicionan cuatro etapas que se mantienen en constate evolución hasta la actualidad. (i) El marketing de relaciones establece como factor fundamental la relación con el cliente; delimitando los siguientes objetivos: desarrollar, potenciar y comercializar relaciones de intercambio con el cliente (Coca, 2008). (ii) La orientación al mercado involucra la necesidad de dirigirse en forma proactiva hacia el exterior de la empresa para mantenerse a largo plazo (Álvarez et al., 2001). Por otra parte, el complemento del (iii) marketing e internet genera (iv), nuevos conceptos que serán discutidos en los apartados posteriores.

\section{Nuevas tendencias del marketing}

En los actuales momentos, el marketing ha tomado un enfoque distante de los fundamentos 
tradicionales. Las nuevas tendencias del marketing no solo se basan en funcionalidades y calidad del producto, sino que se hallan en la búsqueda de brindar experiencias atrayentes y agradables durante la compra, consumo y postconsumo. El marketing experiencial toma esta necesidad y propone como herramienta la generación de emociones y sensaciones consecuentes a la vinculación entre el cliente y la marca ofertante (Hosany \& Witham, 2010).

Schimtt (1999) concluye que el marketing experiencial propone direccionar el foco de atención de la mercadotecnia hacia la necesidad de involucrar al consumidor mediante la interacción emocional producida por la satisfacción que provoca la adquisición del producto o servicio. El punto neurálgico de lo que señala Schimtt se fundamenta en las experiencias de consumo, las cuales son interacciones entre el cliente y producto o empresa, que ocasionan una reacción agradable para el consumidor. Además, son una serie de experiencias personales que implican la participación del individuo y consecuentemente promueven una evaluación de dicha experiencia a través del contraste de las expectativas del cliente y los estímulos postconsumo.

\section{Marketing estratégico}

A mediados de la década de 1980, se plantearon los primeros estudios que demostraron la significancia e importancia de una contribución que abarque las prácticas esenciales de marketing estratégico. El alto interés corporativo de mejorar continuamente la competitividad a nivel de empresa ha fomentado el interés en la mercadotecnia estratégica (Brooksbank \& Taylor, 1999).

Es notable que a partir de ahí se han venido desarrollando investigaciones que han concluido en una serie de documentos y manuscritos con estilo prescriptivo, lo cual representa en términos de economía una ventaja para posicionar el marketing estratégico como una herramienta de carácter obligatorio.

El enfoque comercial actual prioriza los requerimientos del cliente, como base para la creación de productos o servicios que satisfagan las necesidades del consumidor. 
Por tanto, es necesario ejecutar una serie de actividades planificadas de la siguiente forma:

a) Descubrir oportunidades de mercado.

b) Analizar las potencialidades y restricciones del mercado descubierto.

c) Diseñar un producto o servicio acorde a las especificaciones de los potenciales clientes.

d) Evaluar el ciclo de vida del producto o servicio.

e) Conocer a los competidores.

f) Generar una propuesta competitiva y sostenible que represente una ventaja comercial respecto a los competidores (Cravens, 2006).

La estrategia corporativa contempla como factor fundamental el marketing estratégico (Lee, 2004). Por su parte, Coca (2008) aporta información valiosa en este tópico:

En la actualidad se ha posicionado la cercana relación entre la estrategia de marketing y la estrategia corporativa (Vazquez, 1986). el enfoque estratégico propugna por una fuerte dosis de proactividad con el entorno, puesto que, en la actualidad, para el marketing constituye una necesidad esta meta proactiva, por tanto, el marketing puede influir en el entorno (Zeithaml \& Zeithaml, 1984). Se ha buscado constantemente la integración entre el marketing y la planificación estratégica, derivándose en algunos modelos integradores (Wind \& Robertson, 1983).

El plan de marketing estratégico constituye una vía para lograr las metas trazadas de manera óptima y eficiente. A diferencia de la planificación, la estrategia define las pautas para evaluar varias rutas que permitirán, de forma que se obtenga el resultado más adecuado a las necesidades del cliente 0 consumidor.

\subsection{Aplicación del marketing estratégico}

Un caso de estudio interesante es el de China, con una economía que ha emergido como potencia mundial y una industria manufacturera que ocupa la cuarta posición a nivel global; al contrastarse con Estados Unidos y los países europeos, se denota que las empresas e industrias de estos países son más competitivas, debido a que en el país oriental, el marketing estratégico aún 
se encuentra en sus primeras etapas de desarrollo y posicionamiento (Ambler \& Xiucun, 2003).

Existe una relación estadística significativa entre las empresas exitosas y la planificación del marketing, es decir que un adecuado análisis de la situación estratégica genera un mayor rendimiento empresarial; no obstante, este estudio también indica que un 30\% de las empresas de alto desempeño realiza una planificación limitada respecto al presupuesto que se designa para la planificación formal. Este comportamiento refleja que existe una estrecha relación entre las empresas de bajo rendimiento, con una alta gerencia que no practica un análisis integral de la situación estratégica y desarrolla una mala planificación formal del marketing (Huan et al., 2008).

\section{Conclusiones}

El marketing ha experimentado cambios en su conceptualización, partiendo desde la fase de preconceptualización (1900-1959), en la que el marketing se define como aquella parte de la economía que relaciona la producción de un bien o servicio con el consumidor; hasta la conceptualización actual, que se fundamenta en el marketing de relaciones, sensaciones y emociones.

Por otra parte, el marketing se estratifica en dos conceptos importantes para tener en cuenta en un plan de marketing ya que incluyen una etapa analítica (estratégica) y una etapa práctica (operativa).

Por lo tanto, la principal diferencia a resaltar entre el marketing estratégico y el operativo es que la función principal del estratégico es fijar los objetivos a largo plazo mientras que el operativo se basa en acciones concretas a realizar en un plazo medio/corto para lograr los objetivos establecidos. Se analiza el caso puntual de China, que a pesar de ser una potencia mundial aún no desarrolla por completo el marketing estratégico.

\section{Bibliografía}

Álvarez, G., Santos, V., \& Vázquez, C. (2001). El Concepto de Orientación al Mercado: Perspectivas, Modelos $y$ Dimensiones de Análisis. Universidad de Oviedo, Departamento de Administración de Empresas y Contabilidad, Oviedo. 
Ambler, T., \& Xiucun, W. (2003). Measures of marketing success: A comparison between China and the United Kingdom. Asia Pacific Journal of Management, 20(2), 267281.

Arndt, J. (1978). How Broad Should the Marketing Concept Be? Should it be developed into a full-fledged behavioral science? Journal of Marketing, 42(1), 101-103. DOI:

10.1177/0022242978042001 18

Bartels, R. (1988). The History of Marketing Thought.

Publishing Horizons Inc, Columbus.

Bennet, P.D., ed. (1988). Dictionary of Marketing Terms. Chicago: American Marketing Assn.

Brooksbank, R., \& Taylor, D. (2000). Marketing in medium-sized New Zealand companies: The state-of-theart. Journal of International Marketing \& Marketing Research, 25(2), 95-95.
Coca, A. (2008). El concepto de Marketing: pasado $\mathrm{y}$ presente. Revista de Ciencias Sociales, 14(2), 391-414.

Cravens, D. (2006). Strategic marketing's global challenges and opportunities. Handbook of Business Strategy, 7(1), 63-70.

Doi: 10.1108/107757306106186 39

Hosany, S., \& Witham, M. (2010). Dimensions of cruisers' experiences, satisfaction, and intention to recommend. Journal of Travel Research, 49(3), 351-364. DOI: 10.1177/0047287509346859

Huan, G., Brooksbank, R., Taylor, D., \& Babbis, P. (2008). Strategic marketing in Chinese manufacturing companies. Asia Pacific Journal of Marketing and Logistics, 20(3), 276-288. Doi:

$10.1108 / 135558508108900$ 57

Kotler, P. \& Armstrong, G. (2003). Fundamentos de Marketing. 
(6 $6^{\text {a }}$ ed). Prentice Hall, México.

Kotler, P., \& Levy, S. J. (1969). Broadening the concept of marketing. Journal of marketing, 33(1), 10-15. DOI: $10.1177 / 0022242969033001$ 03

Lambin, J. J. \& Peeters, R. (1981). La gestión de Marketing en las empresas. ICE, Madrid.

Lee, Y. I. (2004). Customer service and organizational learning in the context of strategic marketing. Marketing Intelligence \& Planning, 22(6), 652-662.

Doi: $10.1108 / 026345004105590$ 33

McCarthy, E.J. (1964). Basic Marketing: A Managerial Approach. ( (2 $2^{\mathrm{a}}$ ed.). R.D. Irwin, Homewood.

Schmitt, B. H. (1999). Experiential marketing. Free Press, New York.

Vazquez, R. (1986). Desarrollos recientes en la estrategia de marketing. Una recopilación.
Revista Española de Economía, 3(1), 151-171.

Webster Jr, F. (1992). The changing role of marketing in the corporation. Journal of marketing, 56(4), 1-17. Doi: 10.1177/002224299205600 402

West, D. C., Ford, J., \& Ibrahim, E. (2015). Strategic marketing: creating competitive advantage. 3rd edition. Oxford University Press, USA.

Wind, Y., \& Robertson, T. S. (1983). Marketing strategy: new directions for theory and research. Journal of Marketing, 47(2), 12-25. DOI:

$10.1177 / 002224298304700$ 203

Zeithaml, C. P., \& Zeithaml, V. A. (1984). Environmental management: revising the marketing perspective. Journal of Marketing, 48(2), 46-53.

DOI: $10.1177 / 002224298404800$ 204 